\title{
Editorial
}

\section{Annals of Biomedical Engineering 2021 Year in Review}

\author{
Bethany Rowson (iD and Stefan M. Duma \\ Institute for Critical Technology and Applied Science, Virginia Tech, Blacksburg, VA, USA \\ (Received 10 February 2022; accepted 11 February 2022; published online 25 February 2022)
}

Associate Editor Stefan M. Duma oversaw the review of this article.

2021 marked the 49th issue of Annals of Biomedical Engineering (ABME). As the official journal of the Biomedical Engineering Society (BMES), ABME has a broad readership and aims to publish impactful research in all areas of Biomedical Engineering. In 2021, ABME received 956 submissions from 56 countries (Fig. 1). Over the course of the year, 163 articles were accepted, and 278 were published in monthly issues. These issues include both original research and invited review papers, with review papers focusing on timely topics within biomedical engineering. Four special issues were published in 2021: Bioengineering for Women's Health, Concussions in Sports, WIAMan Biomechanics, and Virtual Physiological Human (Fig. 2). These special issues represent areas of growing interest within the BMES community, and are comprised of articles invited by guest editors that are experts within their fields. The following will summarize notable review papers and all special issues published in 2021 .

\section{NOTABLE REVIEW PAPERS}

Bisht et al. reviewed past advancements in bone graft fabrication, and highlighted recent developments in and promise of $3 \mathrm{D}$ printed biomimetic bone grafts. ${ }^{3}$ They summarized the biology of bone, and the difficulties in developing bone graft substitutes. In the past, bone graft substitutes have been composed of metals, biomaterial coated metals, or biodegradable materials. $3 \mathrm{D}$ printing allows for rapid generation of complex

Address correspondence to Bethany Rowson, Institute for Critical Technology and Applied Science, Virginia Tech, Blacksburg, VA, USA. Electronic mail: browson@vt.edu geometries with biocompatible materials and live cells, which could result in fewer adverse outcomes for patients.

Huang and Chalmers reviewed wearable sensors that can be used to monitor solar ultraviolet (UV) exposure in an individual. ${ }^{10} \mathrm{UV}$ radiation is necessary for vitamin $\mathrm{D}$ synthesis in the body, but too much exposure can lead to a number of health problems including eye diseases and skin cancers. Commercial wearable UV sensors could be valuable tools for tracking individual UV exposure so that the user can modify their behaviors for optimal health benefits. However, for optimal use, accuracy of the sensors needs to be verified. Currently available sensors are either photosensitive film-based or electronic.

Masteller et al. reviewed current technologies and recent developments in upper limb neural prostheses. ${ }^{12}$ Realistic sensory feedback systems have not been successfully incorporated into robotic prostheses yet, but would greatly improve the usability and acceptance of the prostheses for amputees. The authors overviewed concepts and technologies involved with sensors used for tactile sensation, techniques for identifying object textures, and methods for relaying sensor data to the user through sensory stimulation.

\section{BIOENGINEERING FOR WOMEN'S HEALTH}

The Bioengineering for Women's Health special issue was published in August of 2021. The issue focused on biomechanics and morphology of female reproductive organs and their supporting structures, and female-related cancers. ${ }^{5}$ The biomechanics of female reproductive organs are unique due to the drastic changes that occur during pregnancy and childbirth. 


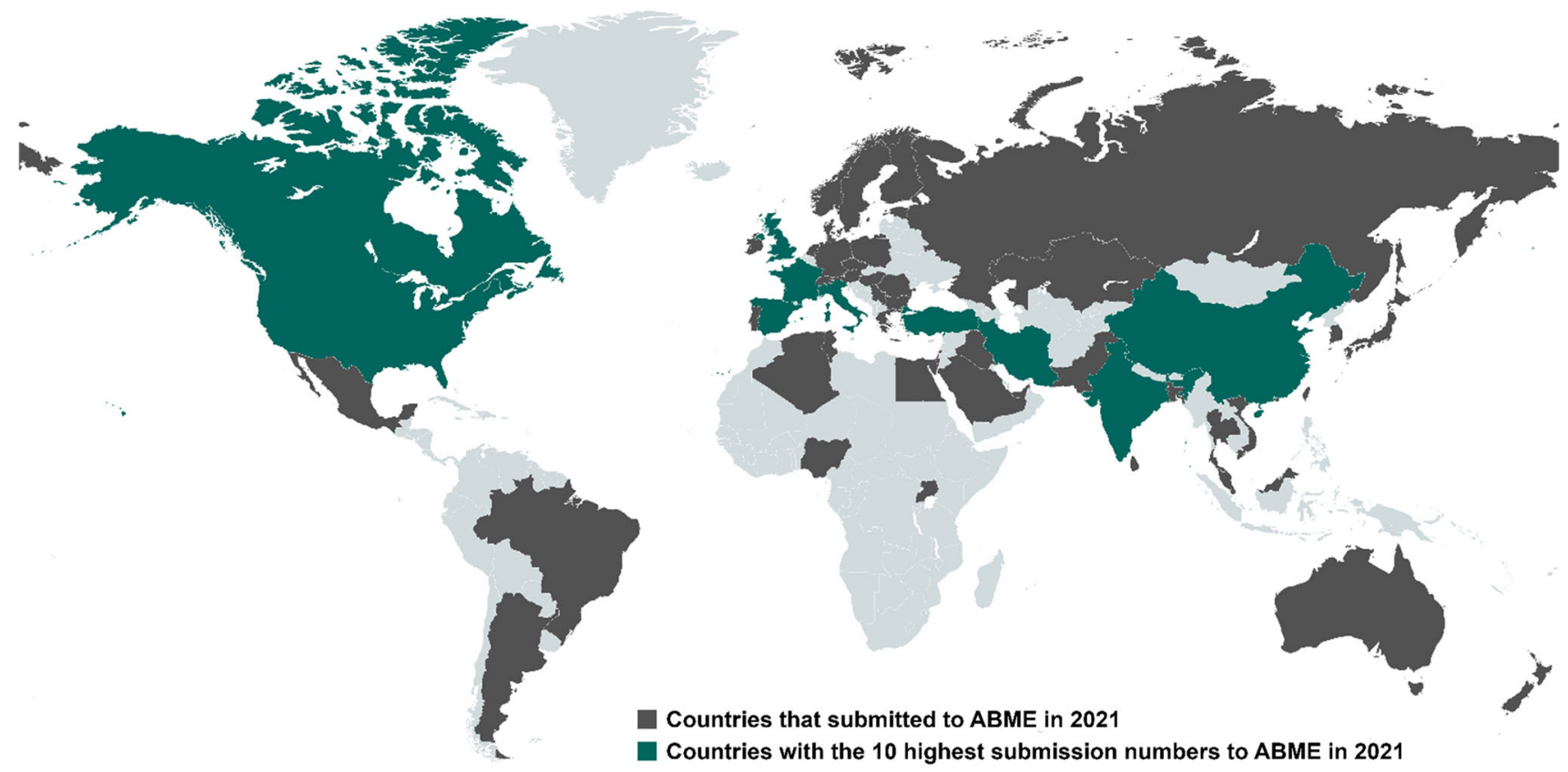

FIGURE 1. Map highlighting countries that submitted manuscripts to ABME in 2021. The countries with the 10 highest submission numbers accounted for over $75 \%$ of all submitted manuscripts.

Grimm reviewed the maternal and clinician-applied forces involved in childbirth. Improved understanding of these forces can be used in computational models to predict maternal and fetal outcomes for childbirth complications. ${ }^{9}$ Characterization of structures that support female pelvic organs are important for understanding disorders such as pelvic organ prolapse. Donaldson et al. reviewed the mechanics of the uterosacral ligaments, which support the uterus and apical vagina, and play an important role in pelvic organ prolapse pathophysiology and treatment. ${ }^{7}$ Pelvic organ prolapse is a prevalent disorder in females with a range of symptoms that substantially decrease quality of life. Beyond the scope of female pelvic organ biomechanics, this special issue also covered new areas of research that may lead to decreased mortality for cancers that disproportionately affect women. ${ }^{1,16}$

\section{CONCUSSIONS IN SPORTS}

In October of 2021, ABME published a special issue on concussions in sports. ${ }^{14}$ This issue was the third in an annual series of special issues focused on concussions in ABME. In the 2021 issue, papers covered a wide range of topics including helmet evaluation for different sports and recreational activities, measure- ment of on-field head impact kinematics, and techniques for brain strain prediction to better understand and prevent head injuries in sports. DeGiacomo et al. compared impact performance of snow sport helmets with rotation-damping systems to a standard snow sport helmet not designed to reduce rotational motion during impact. ${ }^{6}$ The results showed that the rotationdamping systems (MIPS and WaveCel) substantially reduced rotational acceleration, and therefore concussion risk, during impact. Cecchi et al. used instrumented mouthguards to measure head impact kinematics in American football players for prediction of brain strain with a computational model. ${ }^{4}$ Player position and impact direction influenced peak head kinematics and brain strain measurements.

\section{WIAMAN BIOMECHANICS}

The Warrior Injury Assessment Manikin (WIAMan) program was initiated by the US Army in 2010 to develop a specialized anthropomorphic test device (ATD) for simulated underbody blast (UBB) tests. The use of improvised explosive devices (IEDs) has increased in recent military conflicts, and when activated under a vehicle, they transmit high-rate vertical loads to the vehicle occupants. There were previously 


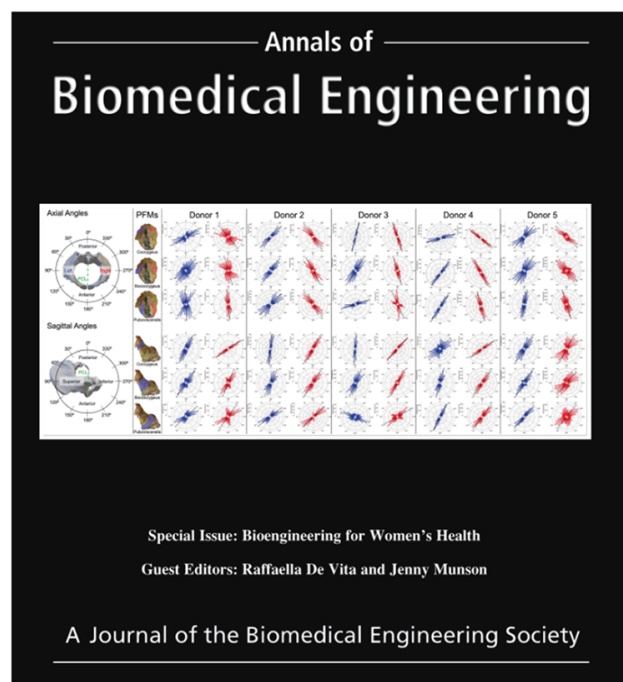

Annals of
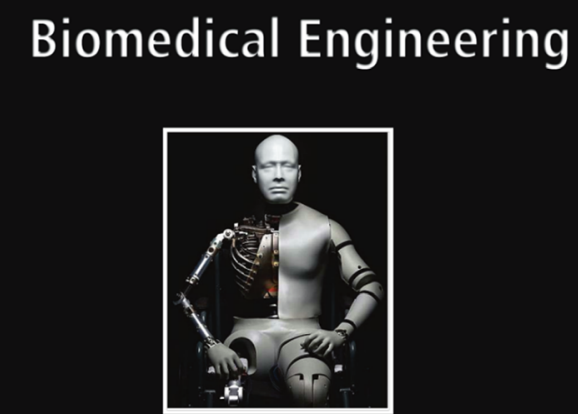

Special Issue: WIAMan Biomechanics

Guest Editors: Catherine M. Carneal, Frank A. Pintar and Narayan Yoganandan

A Journal of the Biomedical Engineering Society

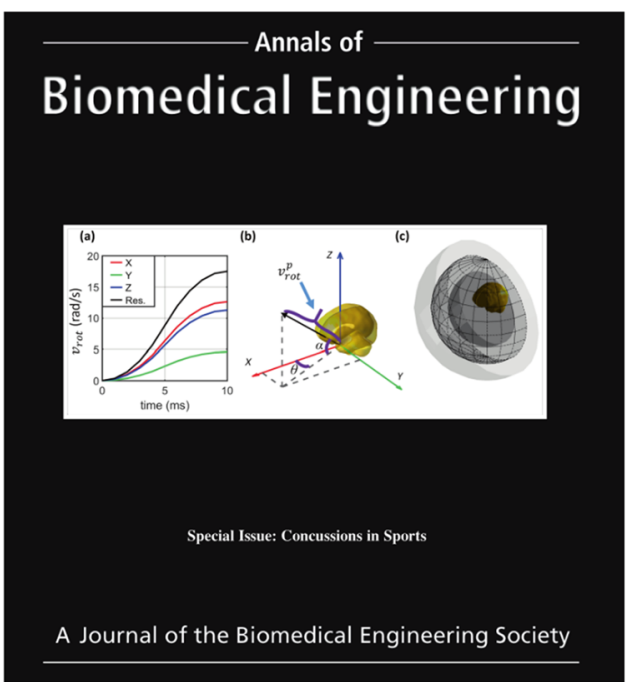

Annals of
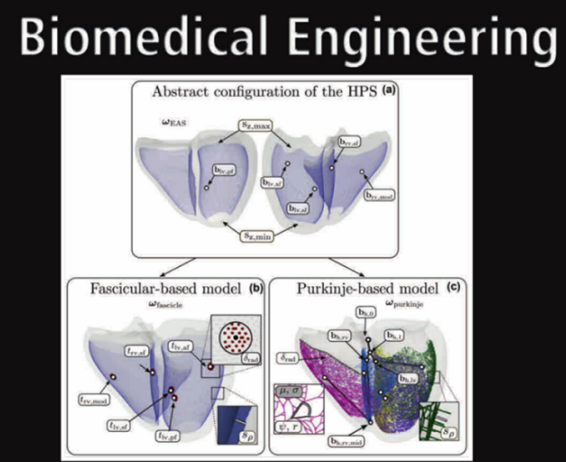

Special Issue: Virtual Physiological Human

Guest Editors: Abdul Barakat, Dominique Chapelle, Irene Vignon-Clementel,

and Joel Stitzel

A Journal of the Biomedical Engineering Society

FIGURE 2. Cover images for all 2021 special issues in ABME. Top left: Bioengineering for Women's Health (August 2021), top right: Concussions in Sports (October 2021), bottom left: WIAMan Biomechanics (November 2021), bottom right: Virtual Physiological Human (December 2021).

no ATDs designed to have a biofidelic response under these conditions, so development of the WIAMan will contribute to better understanding of injury risks and improved protective designs for military Service Members. The November 2021 WIAMan Biomechanics special issue in ABME focused on biofidelity analysis and development of human injury probability curves for the WIAMan. ${ }^{11}$ Many of the studies included in this issue used post mortem human subjects to determine the range of responses for different body regions during UBB and to develop human injury probability curves. ${ }^{15,17,19}$

\section{VIRTUAL PHYSIOLOGICAL HUMAN}

The Virtual Physiological Human special issue was published in December of 2021. The special issue was comprised of selected works that were presented at the Virtual Physiological Human 2020 (VPH2020) conference. This conference focuses on Computational Systems Biomedicine with the goal of translating in silico medicine to practice. The studies selected for the special issue developed or implemented mathematical models to better understand physiological or pathophysiological processes in different body systems. These models were used to evaluate orthopedic devices, ${ }^{8,18}$ noninvasively evaluate hemodynamic indi- 
cators, ${ }^{2}$ and assist with pre-surgical planning ${ }^{13}$ among other applications. The patient-specific and integrated approaches that consider interactions between different physiological systems in an individual are unique to the models presented at VPH2020, and will lead to advancements in in silico medicine.

As ABME approaches its 50th anniversary, our focus remains on publishing impactful research in all areas of interest to our broad biomedical engineeringbased readership. We thank our authors for submitting their important work, and our reviewers for volunteering their time and insight to refine the papers published. The journal would not be possible without these valuable contributions.

\section{REFERENCES}

\footnotetext{
${ }^{1}$ Amirghasemi, F., E. Adjei-Sowah, B. A. Pockaj, and M. Nikkhah. Microengineered 3D tumor models for anticancer drug discovery in female-related cancers. Ann. Biomed. Eng. 49:1943-1972, 2021.

${ }^{2}$ Antonuccio, M. N., A. Mariotti, B. M. Fanni, K. Capellini, C. Capelli, E. Sauvage, and S. Celi. Effects of uncertainty of outlet boundary conditions in a patientspecific case of aortic coarctation. Ann. Biomed. Eng. 49:3494-3507, 2021.

${ }^{3}$ Bisht, B., A. Hope, A. Mukherjee, and M. K. Paul. Advances in the fabrication of scaffold and 3D printing of biomimetic bone graft. Ann. Biomed. Eng. 49:1128-1150, 2021.

${ }^{4}$ Cecchi, N. J., A. G. Domel, Y. Liu, E. Rice, R. Lu, X. Zhan, Z. Zhou, S. J. Raymond, S. Sami, and H. Singh. Identifying factors associated with head impact kinematics and brain strain in high school American football via instrumented mouthguards. Ann. Biomed. Eng. 49:2814 2826, 2021.

${ }^{5}$ De Vita, R., and J. Munson. Special Issue on the Advances in Engineering for Women's Health. New York: Springer, pp. 1785-1787, 2021.

${ }^{6}$ DiGiacomo, G., S. Tsai, and M. Bottlang. Impact performance comparison of advanced snow sport helmets with dedicated rotation-damping systems. Ann. Biomed. Eng. 49:2805-2813, 2021.

${ }^{7}$ Donaldson, K., A. Huntington, and R. De Vita. Mechanics of uterosacral ligaments: current knowledge, existing gaps, and future directions. Ann. Biomed. Eng. 49:1788-1804, 2021.
}

${ }^{8}$ Favre, P., G. Maquer, A. Henderson, D. Hertig, D. Ciric, and $\mathbf{J}$. E. Bischoff. In silico clinical trials in the orthopedic device industry: from fantasy to reality? Ann. Biomed. Eng. 49:3213-3226, 2021.

${ }^{9}$ Grimm, M. J. Forces involved with labor and delivery-a biomechanical perspective. Ann. Biomed. Eng. 49:1819$1835,2021$.

${ }^{10}$ Huang, X., and A. N. Chalmers. Review of wearable and portable sensors for monitoring personal solar UV exposure. Ann. Biomed. Eng. 49:964-978, 2021.

${ }^{11}$ Loftis, K., K. Sandora, and D. Drewry. Introduction to the WIAMan Biomechanics Program. New York: Springer, pp. 2973-2974, 2021.

${ }^{12}$ Masteller, A., S. Sankar, H. B. Kim, K. Ding, X. Liu, and A. H. All. Recent developments in prosthesis sensors, texture recognition, and sensory stimulation for upper limb prostheses. Ann. Biomed. Eng. 49:57-74, 2021.

${ }^{13}$ Narang, H., B. V. Rego, A. H. Khalighi, A. Aly, A. M. Pouch, R. C. Gorman, J. H. Gorman III., and M. S. Sacks. Pre-surgical prediction of ischemic mitral regurgitation recurrence using in vivo mitral valve leaflet strains. Ann. Biomed. Eng. 49:3711-3723, 2021.

${ }^{14}$ Rowson, B., and S. M. Duma. Special Issue on Concussions in Sports. New York: Springer, pp. 2673-2676, 2021.

${ }^{15}$ Rupp, J. D., L. Zaseck, C. S. Miller, A. C. Bonifas, M. P. Reed, D. Sherman, J. M. Cavanaugh, K. Ott, and C. K. Demetropoulos. Whole body PMHS response in injurious experimental accelerative loading events. Ann. Biomed. Eng. 49:3031-3045, 2021.

${ }^{16}$ Schwartz, A. D., A. Adusei, S. Tsegaye, C. A. Moskaluk, S. S. Schneider, M. O. Platt, D. Seifu, S. R. Peyton, and C. C. Babbitt. Genetic mutations associated with hormone-positive breast cancer in a small cohort of Ethiopian women. Ann. Biomed. Eng. 49:1900-1908, 2021.

${ }^{17}$ Sherman, D., K. Somasundaram, P. Begeman, S. Foley, J. Greb, C. Bir, C. K. Demetropoulos, and J. M. Cavanaugh. Dynamic response of the thoracolumbar and sacral spine to simulated underbody blast loading in whole body post mortem human subject tests. Ann. Biomed. Eng. 49:30463079, 2021.

${ }^{18}$ Viceconti, M., C. Curreli, F. Bottin, and G. Davico. Effect of suboptimal neuromuscular control on the risk of massive wear in total knee replacement. Ann. Biomed. Eng. 49:33493355, 2021.

${ }^{19}$ Voo, L., K. Ott, T. Metzger, A. Merkle, and D. Drewry. Severe calcaneus injury probability curves due to underbody blast. Ann. Biomed. Eng. 49:3118-3127, 2021.

Publisher's Note Springer Nature remains neutral with regard to jurisdictional claims in published maps and institutional affiliations. 